\title{
Influence of State and/or Trait Anxieties of Wistar Rats in an Anxiety Paradigm
}

\author{
Rashmi Madhava Rao Monika Sadananda \\ Brain Research Laboratory, Biotechnology Division, Department of Biosciences, Mangalore University, \\ Mangalagangothri, Mangalore, India
}

\section{Key Words}

Elevated plus maze - Restraint - Stress · Population .

Behavior

\begin{abstract}
Systematic individual differences between male Wistar rats can be detected in paradigms such as the elevated plus maze (EPM), which is a widely used behavioral paradigm that measures fear-motivated avoidance behavior. It has been extensively used to assess anxiety profiles with face, construct and predictive validities. During a typical EPM test, animals actively avoid the open arms in favour of the closed arms. We investigated whether individuals carry inherent trait anxiety profiles and whether perturbations of different intensities influence anxiety measures. Inherent anxiety levels and coping strategies following stress have become critical determinants in pre-disposition to other neuropsychiatric disorders and affect biomedical interventions in individuals. One group of rats was screened on EPM and in the activity box. Another set of rats were randomly divided into groups and subjected to perturbations of acute and subchronic isolation or restraint and tested in the EPM. Based on open-arm time in the EPM, low or high anxiety profiles were identified with significant differences in all measures. Perturbations of different intensities induced differential anxiety measures as expressed in the EPM. Anxiety levels were sig-
\end{abstract}

nificantly reduced in sub-chronic restrained subjects, while isolation did not show marked difference. Anxiety profiles become evident from broad sample sizes and could constitute a critical limiting factor in personalized treatments. Stress-induced anxiety disorders could implicate comorbidity to other neuropsychiatric disorders in individuals. Coping strategies come to the fore in repeated sub-chronic perturbations indicating adaptive responses to the stressor, while acute perturbation enhances expression of anxiety behaviors.

(c) 2016 S. Karger AG, Basel

\section{Introduction}

The elevated plus maze (EPM) is the most widely used behavioral paradigm that measures fear-motivated avoidance behavior in rodents. It is based on the conflict between the animal's innate behavioral urge to explore novel spaces and its fear of open, brightly lit spaces; in other words, it assays approach and avoidance. This test has been validated using behavioral, physiological and pharmacological approaches. Anxiolytic drugs increase the time spent on the open arms; hence, rats that avoid open spaces (arms) to a greater extent are said to be more anxious. The principal construct here is that movements are cautious in neophobia, while neophilia is characterized

\section{KARGER}

E-Mail karger@karger.com

www.karger.com/aon
(C) 2016 S. Karger AG, Basel

0972-7531/16/0231-0044\$39.50/0
Monika Sadananda

Department of Biosciences

Mangalore University

Mangalagangothri, Karnataka 574199 (India)

E-Mail monikasadananda@gmail.com 
by ample locomotion and inspection or exploration. EPM thus is an unconditioned test for anxiety that demonstrates face, construct and predictive validities of the anxiety model. However, it can be used only once as it demonstrates an one-trial tolerance effect or test decay on second exposure [1].

Work with selectively bred and outbred Wistar rats [2, 3] based on the percentage of open-arm time in the EPM has shown that male adult Wistar rats demonstrate either low or high anxiety-like behavior. This 'trait' has been reported to remain stable under re-test conditions (in nonEPM paradigms) and is correlated with other behaviors where anxiety plays a role, such as in object burying and avoidance learning $[4,5]$. This behavioral trait of high or low anxiety is also manifested in the emission of ultrasonic vocalizations and in striatal serotonin and cytokine levels [6].

Any stimulus that interferes with brain homeostasis acts as a stressor and evokes conservation or withdrawal as a behavioral response. Re-establishment of homeostasis is achieved by evoking adaptive responses leading to a coping strategy [7]. Coping mechanisms have an adaptive value in a stressful situation [8] and could induce resilience to stress. The elicited responses may differ depending on the type of stress, sex, age and hormonal state of the individual. The limbic hypothalamic-pituitary-adrenal (HPA) axis is the primary circuit that is involved in initiation, regulation and termination of a stress response, with the brain areas involved in stress control and response being similar [9]. Earlier studies have shown behavioral changes after exposure to inescapable stress such as restraint [10].

For a better understanding of state and trait anxieties, the EPM was used with certain perturbations that could influence expression of anxiety-like behaviors. Our aim was to ascertain whether rats identical in strain, sex and age differ systematically in anxiety-like behavior. The second aim was to test whether anxiety traits are also manifested in other behavioral paradigms, such as a novel activity box. Finally, we used acute and sub-chronic pre-test perturbations to observe how 'state' or induced anxiety impacts anxiety behaviors.

\section{Methods}

\section{Subjects}

Young adult male outbred Wistar rats (Sri Venkateshwara Enterprises, Bangalore), weighing $137.84 \pm 2.42 \mathrm{~g}(\mathrm{n}=65)$ at the beginning of the experiment were used. They were housed in groups of 4 in polypropylene cages under standard laboratory conditions with food and water ad libitum. The housing room was maintained on a 12-hour light/dark cycle. Ambient temperature was between 25 and $27^{\circ} \mathrm{C}$. All animals were handled on 3 consecutive days before the experiments began. All experiments were conducted in the light cycle (9:00-17:00 h) in accordance with the ethical regulations for animal experimentation laid down by CPCSEA and cleared by the Institutional Animal Ethics Committee (SAC/ IAEC/105/2011) of the host institution. All measures were taken to minimize pain and discomfort, other than that critical to the experiment.

\section{Elevated Plus-Maze}

The EPM apparatus was made of acrylic (black) and consisted of 2 open arms $(50 \times 10 \mathrm{~cm})$ and 2 closed arms with no roof $(50 \times$ $10 \times 40 \mathrm{~cm})$ at right angles to each other and an open square $(10 \times 10 \mathrm{~cm})$ in the center. The maze was elevated $50 \mathrm{~cm}$ above the floor. The animals were placed into the center, facing the same open arm each time. The maze was cleaned thoroughly with $1 \%$ acetic acid before the next animal was introduced. If an animal fell from the maze, it was immediately placed back in the position from which it had fallen. The EPM recording was of 5 min duration.

\section{Activity Box}

The activity box consists of an open cube of dimensions $40 \times$ $40 \times 40 \mathrm{~cm}$, placed at an elevation of $50 \mathrm{~cm}$ above the ground. The activity box was cleaned thoroughly with $1 \%$ acetic acid before the next animal was introduced. The recording was of 5 min duration.

\section{Overt Behavior Recording and Analysis}

Behavior was recorded using a Panasonic CCD camera fed to a Piccolo frame grabber card and analyzed using a tracking and video recording software, Ethovision ${ }^{\circledR}$ XT version 8.0 (Noldus, Netherlands). Zones were marked and a template was created to digitize physical distances. The following zones were defined: for activity box - center versus periphery; for EPM - open arms, closed arms and center. An entry was defined as the 4 paws of the subject being inside the template zone. Rearings were scored by a person blind to the experimental conditions. All other measures listed below were software generated, such as duration, frequency, latency, distance moved in the activity box and EPM.

\section{Perturbations}

Another set of rats were then randomly divided into 5 groups: (1) group-held controls; (2) $2 \mathrm{~h}$ of isolation in bare new cage (acute); (3) $2 \mathrm{~h}$ of physical restraint in restrainer (acute); (4) $2 \mathrm{~h}$ of isolation for 5 consecutive days (sub-chronic) and (5) $2 \mathrm{~h}$ of physical restraint for 5 consecutive days (sub-chronic). Restraining was done using $20 \times 6.3 \mathrm{~cm}$ transparent cylindrical tube procured from Orchid Scientifics, Nashik, India. One end of it was enclosed with the provision for breathing. The other end was clamped using a cylindrical disc. The bottom had a slit allowing the animal to urinate and excrete freely. After the perturbations, the animals were placed in the EPM, recorded and analyzed as described above.

\section{Statistical Analyses}

All results are expressed as mean \pm SEM. The $t$ test or one-way analysis of variance (ANOVA) with Tukey's post hoc tests was performed. The level of significance was defined as $\mathrm{p} \leq 0.05$. 
Fig. 1. a Scatter plot of open arm time on the EPM. Open-arm times of all animals were plotted serially. The dividing line indicates the median split used to classify the rats into 2 groups based on open-arm time into HA and LA groups. b Anxiety index of LA group was significantly lower than HA group. Anxiety index was calculated as: 1 ((open-arm time/total time) + (open-arm entries/total entries))/2. The read-out of the anxiety index correlates with the openarm times. The whiskers of the box plot represent minimum to maximum. ${ }^{* * *} \mathrm{p}<$ 0.001 .

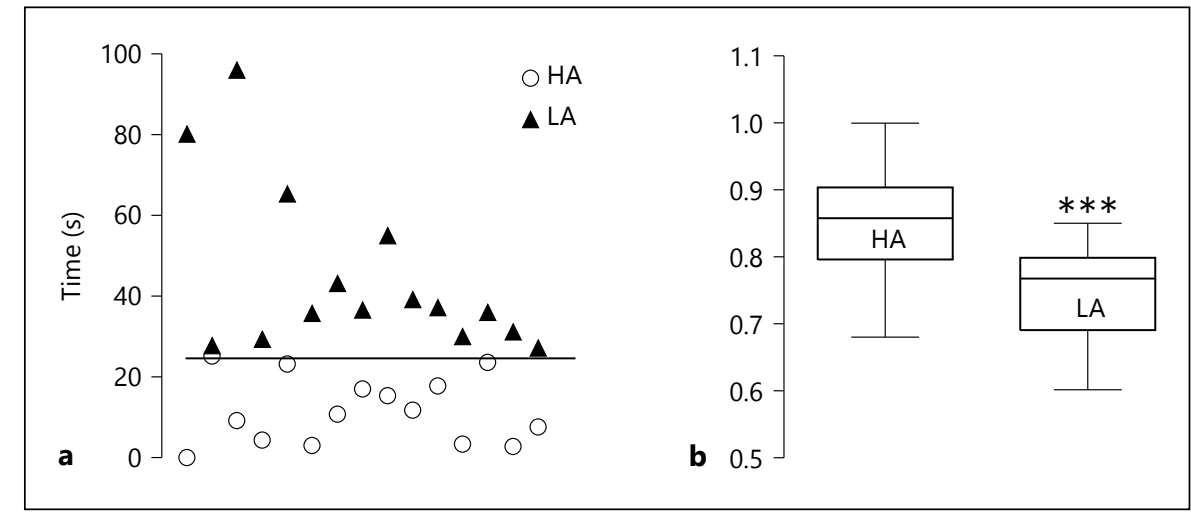

Table 1. EPM measures - HA vs. LA

\begin{tabular}{|c|c|c|c|c|}
\hline Variable & Zone & HA & LA & Statistic, $\mathrm{p}$ value \\
\hline Duration, $s$ & $\begin{array}{l}\text { Open arm } \\
\text { Closed arm } \\
\text { Centre }\end{array}$ & $\begin{array}{r}11.68 \pm 2.15 \\
255.44 \pm 6.71 \\
41.62 \pm 6.72\end{array}$ & $\begin{array}{r}44.68 \pm 5.33^{* * *} \\
211.21 \pm 8.38^{* * *} \\
69.34 \pm 8.77^{*}\end{array}$ & $\begin{array}{l}\mathrm{t}_{28}=5.74, \mathrm{p}=0.001 \\
\mathrm{t}_{28}=4.12, \mathrm{p}=0.001 \\
\mathrm{t}_{28}=2.50, \mathrm{p}=0.018\end{array}$ \\
\hline Frequency, $\mathrm{n}$ & $\begin{array}{l}\text { Open arm } \\
\text { Closed arm }\end{array}$ & $\begin{array}{r}3.6 \pm 0.63 \\
10.13 \pm 1.4\end{array}$ & $\begin{array}{l}6.33 \pm 0.57^{* *} \\
11.8 \pm 1.05\end{array}$ & $\begin{array}{l}\mathrm{t}_{28}=3.20, \mathrm{p}=0.003 \\
\mathrm{t}_{28}=0.95, \mathrm{p}=0.349\end{array}$ \\
\hline Latency, s & Open arm & $26.46 \pm 6.43$ & $8.72 \pm 2.66^{*}$ & $\mathrm{t}_{28}=2.54, \mathrm{p}=0.016$ \\
\hline
\end{tabular}

\section{Results}

Subjects demonstrated great variation in open arm times. A scatter plot of open-arm times across all animals showed individual differences from no time spent in the open arm to close to one-third of the entire testing period spent on the open arm. Based on the time spent in the open arms, animals could be classified into 2 groups using a median split: (1) high anxious (HA) or low open-arm time and (2) low anxious (LA) or high open-arm time.

LA animals showed an increased open-arm time compared to the HA group (fig. 1a) which was highly significant $(t=5.74$; d.f. $=28 ; \mathrm{p}<0.001)$. The converse was true of closed-arm time $(\mathrm{p}<0.001)$. Latency to first entry of an open arm was 3 times more in HA animals (26.46 $\pm 6.43 \mathrm{~s}$ ) than in the LA group $(8.72 \pm 2.66 \mathrm{~s})$, which was significant $(\mathrm{p}<0.05)$. HA animals spent $85 \%$ of the entire testing period in the closed arm when compared to the LA animals that spent $70 \%$, which was highly significant $(\mathrm{p}<0.001)$. Open-arm entries of LA animals were twice as much as those of HA animals, which was very significant ( $\mathrm{p}<$ 0.01 ). Time spent in the center of the EPM of HA animals was significantly $(\mathrm{p}<0.05)$ lower than in LA animals. Number of entries into the closed arm was comparable between HA and LA rats. The mean \pm SEM values of all parameters are given in table 1.

When open-arm time and open-arm entries are taken in relation to total number of entries (both arms) and total time spent on the EPM, an anxiety index or measure ranging from 0 to 1 is obtained (fig. 1b). Here, anxiety measures range from 0.6 to 1.0 , with $\mathrm{HA}$ and LA rats showing significant differences in anxiety levels $(\mathrm{t}=4.043$; d.f. $=28 ; \mathrm{p}=0.0004)$.

The novel activity box paradigm returned no significant differences in ambulation or exploratory behavior between HA and LA animals. Specifically, measures (distance moved, center entries, rearing frequency) yielded no significant differences between HA and LA rats. Time spent in the center was marginally increased in LA animals, with a corresponding marginal decrease in periphery time, while the reverse was observed for HA animals. These data however did not reach significance $(p=0.09)$. The mean \pm SEM values of all measures are given in table 2. 
Fig. 2. Pre-test perturbation-induced openarm times on EPM: control vs. acute isolation/restraint and sub-chronic isolation/ restraint. Iso- $2=$ Isolation for $2 \mathrm{~h}$, Res- $2=$ restraint for $2 \mathrm{~h}$, Iso- $2 \times 5=$ isolation of $2 \mathrm{~h}$ on 5 consecutive days, Res- $2 \times 5=$ restraint of $2 \mathrm{~h}$ on 5 consecutive days. ${ }^{*} \mathrm{p}<0.05$; ${ }^{* *} \mathrm{p}<0.01{ }^{* * *} \mathrm{p}<0.001$.

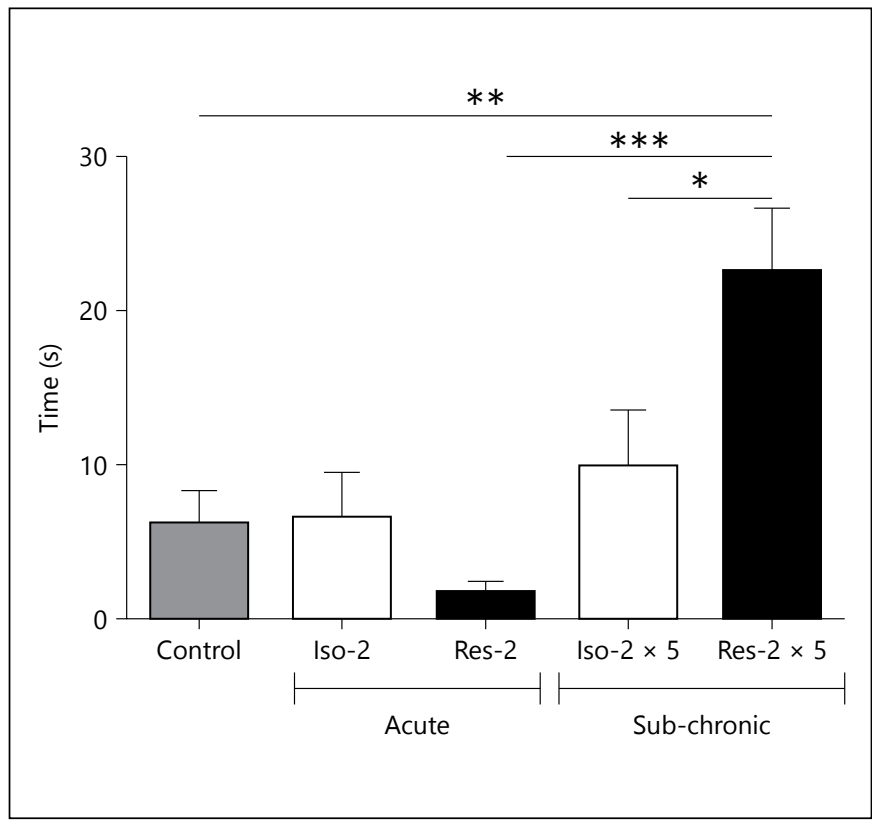

Table 2. Activity box measures in HA/LA rats

\begin{tabular}{llccl}
\hline Variable & Zone & HA & LA & Statistic, $\mathrm{p}$ value \\
\hline Duration, $\mathrm{s}$ & Center & $5.96 \pm 1.45$ & $11.24 \pm 2.73$ & $\mathrm{t}_{17}=1.75, \mathrm{p}=0.097$ \\
& Periphery & $294.2 \pm 1.45$ & $289 \pm 2.73$ & $\mathrm{t}_{17}=1.75, \mathrm{p}=0.097$ \\
\hline Frequency, $\mathrm{n}$ & Center & $6.18 \pm 1.41$ & $6.77 \pm 1.48$ & $\mathrm{t}_{18}=0.28, \mathrm{p}=0.776$ \\
\hline Ambulation, cm & Center + periphery & $1,293 \pm 107.9$ & $1,209 \pm 98.25$ & $\mathrm{t}_{16}=0.56, \mathrm{p}=0.583$ \\
\hline Exploration, $\mathrm{n}$ & Center + periphery & $21.45 \pm 2.98$ & $20.56 \pm 2.53$ & $\mathrm{t}_{18}=0.22, \mathrm{p}=0.825$ \\
\hline
\end{tabular}

HA vs. LA rats showed no significant differences in any of the variables measured.

Physical restraint for $2 \mathrm{~h}$ (acute) induced a decrease in open-arm time (1.68 $\pm 0.68 \mathrm{~s})$ when compared to controls $(6.25 \pm 2.22 \mathrm{~s})$ and time-matched isolates $(6.71 \pm 2.96 \mathrm{~s})$. Latency to enter the open arm was significantly increased for acute restrained animals thus demonstrating a first open-arm entry after $177.8 \pm 42.49 \mathrm{~s}$, which is after half of the test time when compared to controls $(14.71 \pm 7.27$ $\mathrm{s} ; \mathrm{p}<0.001)$ and the time-matched isolates $(30.36 \pm 17.1$ $\mathrm{s} ; \mathrm{p}<0.01)$. Acute physical restraint of $2 \mathrm{~h}$ induced a significant decrease in ambulation with differences emerging with the controls $(\mathrm{p}<0.01)$ and with the time-matched isolates $(\mathrm{p}<0.05)$. This was expressed also in the significantly reduced closed-arm entries when compared to controls ( $\mathrm{p}<0.01)$, though it was not different from timematched isolates.

Influence of State and/or Trait Anxieties of Wistar Rats in an Anxiety Paradigm
In the sub-chronic group that underwent $2 \mathrm{~h}$ of physical restraining on 5 consecutive days, open-arm time was increased. A one-way ANOVA returned highly significant differences across groups $\left(\mathrm{F}_{4,30}=6.14, \mathrm{p}=0.001\right.$; fig. 2). When compared to time-matched isolates, it was significantly $(\mathrm{p}<0.05)$ increased. Ambulation was increased in the sub-chronic restrained group when compared to time-matched isolates, but did not reach significance (table 3).

All the perturbations, whether acute or sub-chronic restraint and time-matched isolations induced a reduction in ambulation, which also translated into a significant reduction $\left(\mathrm{F}_{4,30}=4.85, \mathrm{p}=0.002\right)$ in closed-arm entries. When compared to controls, closed-arm entries were very significantly reduced $(\mathrm{p}<0.01)$ in acute re- 
Table 3. Comparative measures of anxiety behaviors following pre-test perturbations: control; acute $-2 \mathrm{~h}$ isolation or restraint and subchronic isolation and restraint. $\mathrm{n}=7$ in each group

\begin{tabular}{|c|c|c|c|c|c|}
\hline \multirow[t]{2}{*}{ Variable } & \multirow[t]{2}{*}{ Control } & \multicolumn{2}{|l|}{ Acute } & \multicolumn{2}{|l|}{ Sub-chronic } \\
\hline & & isolation $(2 \mathrm{~h})$ & restraint $(2 \mathrm{~h})$ & isolation $(2 \mathrm{~h} \times 5)$ & restraint $(2 \mathrm{~h} \times 5)$ \\
\hline OA time, s & $6.25 \pm 2.22$ & $6.71 \pm 2.96$ & $1.68 \pm 0.68$ & $9.90 \pm 3.64$ & 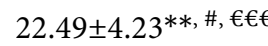 \\
\hline CA time, s & $267.8 \pm 7.03$ & $263.1 \pm 8.67$ & $280.2 \pm 5.2$ & $262.6 \pm 10.51$ & $254.2 \pm 8.5$ \\
\hline Centre time, $s$ & $25.78 \pm 5.14$ & $40.96 \pm 8.7$ & $20.02 \pm 5.2$ & $30.28 \pm 7.82$ & $23.80 \pm 5.01$ \\
\hline OA latency, s & $14.71 \pm 7.27$ & $30.36 \pm 17.1$ & $177.8 \pm 42.49^{* * *, \# \#}$ & $51.98 \pm 26.67$ & $45.48 \pm 21.37^{€}$ \\
\hline OA entries, $\mathrm{n}$ & $2 \pm 0.68$ & $1.25 \pm 0.45$ & $1.12 \pm 0.58$ & $2.7 \pm 0.77$ & $3.33 \pm 0.79$ \\
\hline CA entries, $n$ & $15 \pm 1.03$ & $8.45 \pm 1.47^{*}$ & $6.37 \pm 1.17^{* *}$ & $8.55 \pm 1.57^{*}$ & $8.44 \pm 2.02 *$ \\
\hline Dist moved, $\mathrm{cm}$ & $1,568 \pm 91.46$ & $1,458 \pm 138.5$ & $946.3 \pm 123.1^{* * \text {, \# }}$ & $976.2 \pm 148.9^{* *}$ & $1,325 \pm 115.1$ \\
\hline Anxiety index & $0.91 \pm 0.02$ & $0.89 \pm 0.03$ & $0.94 \pm 0.02$ & $0.89 \pm 0.02$ & $0.81 \pm 0.03^{€}$ \\
\hline
\end{tabular}

* Indicates significant differences between all perturbed groups vs. control. \# Denotes significant differences in acute condition between isolates and restrained animals and in sub-chronic condition between isolates and restrained animals. ${ }^{€}$ Indicates significant differences in sub-chronic restraint when compared to acute restraint. ${ }^{*, \#, €} \mathrm{p}<0.05 ;{ }^{* *}, \#$ p $<0.01$; $^{* * *}, € € \mathrm{p}<0.001$.

straint, while they were significantly reduced in subchronic restraint and both the isolate groups $(\mathrm{p}<0.05)$. Latency to enter the open arm was also significantly different $\left(\mathrm{F}_{4,30}=6.36, \mathrm{p}=0.001\right)$, with post hoc tests returning a highly significant increase in the acute restrained group only when compared to controls $(\mathrm{p}<0.001)$ and when compared to their time-matched isolates $(\mathrm{p}<0.01)$.

When comparing acute with sub-chronic restraint, the sub-chronic restraint group demonstrated an increased open-arm time that was highly significant ( $\mathrm{p}<0.001)$. Latency to enter open arm was also significantly reduced $(\mathrm{p}<0.05)$ when compared to the acute restrained group. No other parameters were affected as a result of repeated restraint.

The anxiety index parameter was significantly increased $(\mathrm{p}<0.05)$ in acute restraint when compared to repeated restraint. The mean \pm SEM and all other parameters are given in table 3.

\section{Discussion}

In recent years, research conducted at behavioral, physiological, biomedical applicational and interventional levels are all taking the individual into account as innumerable studies [11] have demonstrated individual differences across species including humans. Individual differences in behavior are determined both by genetic variances and by events in the surroundings that influence an individual's anxiety levels. Individual differences seen in stress physiology and behavioral profiles could deter- mine how each individual copes with stress, a critical factor in biomedicine [8]. Research in this direction will expose building blocks of affect-related personalities and the differences in them, opening up plausible strategies towards personalized treatments, by revealing substrates for vulnerability. The differences in these substrates appear to cause inter-individual differences, giving way to variability in susceptibility to various psychiatric disorders. Neurobiological foundations to these affective disorders may help in determining if a particular drug or treatment will be useful for a given individual or not. As invasive analyses are not possible with human subjects, animal studies of affective neuroscience are on the rise [12].

Decreased open-arm time in the EPM is often correlated with freezing behaviors or immobility that index anxiety levels. All measures are based on the principle of developing an avoidance approach conflict and indicate whether the animal follows its innate urge to explore new spaces, that is, the open arms or its fear of elevated, open spaces. It is to be expected that individuals in a population vary, but that it can also be obtained in a single randomized trial, as we have shown here, which has implications in humans, as individual differences could predict predisposition to affective disorders [13]. These individual differences are apparent when faced with environmental challenges or stressful situations like a novel environment [11]. Consistent individual differences have been seen even at the physiological level [14]. Preliminary studies indicate that HA-LA rats [15] also demonstrate subtle differences in memory retention of spatial information when tested in the radial arm maze [16]. 
That pre-test manipulations induce different anxiety profiles when tested in adolescents and adults have been shown in SD rats [17]. Studies show that individually housed rats were hyper-reactive in novel environments and showed slower habituation at all time points tested $[18,19]$ though the isolates tested here were only socially isolated for the duration of the experiment. Different periods of social isolation also have short- and long-term influences on behavior. However, all these isolation periods were for longer durations than the brief period of $2 \mathrm{~h}$ used here.

Restraint stress increases anxiety-like behavior. Activity box measures were not taken here as various other studies have shown that restraint stress reduces locomotor activity [20]. Adolescent rats demonstrated increased anxiety in the light-dark box following restraint when compared to adults [21]. The differences in behavior induced by acute and sub-chronic restraint correlated with corticosterone levels (unpublished data), while other studies show concomitant plasma ACTH levels [22], with increase from acute restraint and a decrease after the third day of restraint. The latter study showed a decrease in open arm entries and time following acute restraint (15 $\mathrm{min}, 30 \mathrm{~min}, 1 \mathrm{~h}$ ), but also at a sub-chronic level of 3 days, which is at variance from what was observed in this study. Possibly, 3 days was not sufficient to increase activity on the open arms. Moreover, the restraint period used in the latter study was for $1 \mathrm{~h}$ only.

The behavioral differences in the acute and sub-chronic groups could also be due to differences between escapable and inescapable stress [7]. Acute restraint, which is inescapable and by itself anxiogenic, leads to increased anxiety in the EPM [23]. However, during 5 consecutive exposures, the situation already involves a cognitive appraisal and, therefore, does not induce the state anxiety observed in the first exposure [24]. Serotonin levels are also increased after $1 \mathrm{~h}$ of restraint, more so in the hypothalamus, indicating the activation of the HPA axis again [25]. The difference in state anxiety in acute and sub-chronic exposures could be explored further by the use of anxiolytics.

Here, previous, repeated exposure to the same stressor evoked a different response with repeated sub-chronic restraint having no effect on anxiety behaviors. Whether it is because of repeated handling in the days preceding the test remains to be seen, however other studies indicate that this is not the case [1]. Prior exposure to a novel environment is said to increase motor activity in the EPM and could have translated into the increased entries into the open arm observed here [1]. Moreover, aversiveness of the test condition and prior exposure are important factors that may influence the expression of anxiety.
Genetic pre-disposition in combination with environmental risk factors contribute toward susceptibility to affective disorders. Thus, measures of individual anxiety levels can facilitate early detection of a psychiatric disorder [12]. Persistence and co-segregation of a trait validates the presence of a phenotype and are among the criteria used in a selection strategy based model of interindividual difference. Persistence of the endophenotype, even under environmental changes, further substantiates the presence of that trait.

Behavioral differences do not affect survival. If the variation is neutral, random or non-adaptive, those differences are maintained without having major effects. However, neophobhic individuals were found to have shorter lifespan compared to neophilics [26]. The present study assessing differences in behavioral responses due to inherent anxiety levels or 'trait' and induced anxiety through acute and sub-chronic exposures, that is 'state' anxiety, suggests that inter-individual differences could possibly be present even at structural and functional level, reflected in personalities and coping styles owing to endophenotypes and interactions with adverse events. This could help assess vulnerability to psychiatric disorders and help evolve perspectives and interventions in psychopharmacology.

\section{Author Contributions}

R.M.R. carried out the experiment and did the analysis and graphs. R.M.R. and M.S. wrote the paper.

\section{Acknowledgements}

Supported by the DST under the Cognitive Science Research Initiative (SR/CSI/05/2009). This work was carried out in the corresponding author's former lab. Preliminary results of this study were presented at the International Symposium on Recent Trends in Neurosciences held at DIPAS-DRDO, New Delhi from October 30, 2011 to November 1, 2011.

\footnotetext{
References $\quad 1$ Walf AA, Frye CA: The use of the elevated plus maze as an assay of anxiety-related behavior in rodents. Nat Protoc 2007;2:322-328.

2 Driscoll P: Hypothalamus serotonin turnover in rat lines selectively bread for differences in active, two-way avoidance behavior. Adv Biosci 1988;70:55-57.

3 Driscoll P, Dedek J, Martin JR, et al: Regional 5-HT analysis in Roman high- and low-avoidance rats following MAO inhibition. Eur J Pharmacol 1980;68:373-376.
}

Influence of State and/or Trait Anxieties of Wistar Rats in an Anxiety Paradigm
Ann Neurosci 2016;23:44-50

DOI: $10.1159 / 000443555$ 
4 Ho YJ, Eichendorff J, Schwarting RK: Individual response profiles of male Wistar rats in animal models for anxiety and depression. Behav Brain Res 2002;136:1-12.

5 Ho YJ, Pawlak CR, Guo L, et al: Acute and long-term consequences of single MDMA administration in relation to individual anxiety levels in the rat. Behav Brain Res 2004;149: 135-144.

6 Pawlak CR, Ho YJ, Schwarting RK, et al: Relationship between striatal levels of interleukin-2 mRNA and plus-maze behaviour in the rat. Neurosci Lett 2003;341:205-208.

7 Koolhaas JM, Bartolomucci A, Buwalda B, et al: Stress revisited: a critical evaluation of the stress concept. Neurosci Biobehav Rev 2011; 35:1291-1301.

8 Carere C, Caramaschi D, Fawcett TW: Covariation between personalities and individual differences in coping with stress: converging evidence and hypotheses. Curr Zool 2010; 56:728-740.

9 Stankiewicz AM, Swiergiel AH, Lisowski P: Epigenetics of stress adaptations in the brain. Brain Res Bull 2013;98:76-92.

10 Amat J, Sparks PD, Matus-Amat P, et al: The role of the habenular complex in the elevation of dorsal raphe nucleus serotonin and the changes in the behavioral responses produced by uncontrollable stress. Brain Res 2001;917: 118-126.
11 Pawlak CR, Ho YJ, Schwarting RK: Animal models of human psychopathology based on individual differences in novelty-seeking and anxiety. Neurosci Biobehav Rev 2008;32: 1544-1568.

12 Harro J: Inter-individual differences in neurobiology as vulnerability factors for affective disorders: implications for psychopharmacology. Pharmacol Ther 2010;125:402-422.

13 Hariri AR: The neurobiology of individual differences in complex behavioral traits. Annu Rev Neurosci 2009;32:225-247.

14 Mora F, Segovia G, del Arco A, et al: Stress, neurotransmitters, corticosterone and bodybrain integration. Brain Res 2012;1476:71-85.

15 Rao RM, Sadananda M: Anxiety-motivated avoidance behaviour in the elevated plus maze: a trait and state study in Wistar rats. Int Symp Recent Trends Neurosci 2011:149-150.

16 Rao RM, Sadananda M: Cognition and neurochemistry in a state-trait anxiety paradigm in Wistar rats. Proc Cogn Cell Syst 2014:2930.

17 Doremus-Fitzwater TL, Varlinskaya EI, Spear LP: Effects of pretest manipulation on elevated plus-maze behavior in adolescent and adult male and female Sprague-Dawley rats. Pharmacol Biochem Behav 2009;92:413-423.

18 Gentsch C, Lichtsteiner M, Feer H: Behavioural comparisons between individuallyand group-housed male rats: effects of novel environments and diurnal rhythm. Behav Brain Res 1982;6:93-100.
19 Paulus MP, Bakshi VP, Geyer MA: Isolation rearing affects sequential organization of motor behavior in post-pubertal but not pre-pubertal Lister and Sprague-Dawley rats. Behav Brain Res 1998;94:271-280.

20 Klenerova V, Flegel M, Skopek P, et al: Galanin modulating effect on restraint stressinduced short- and long-term behavioral changes in Wistar rats. Neurosci Lett 2011; 502:147-151.

21 Slawecki CJ: Comparison of anxiety-like behavior in adolescent and adult Sprague-Dawley rats. Behav Neurosci 2005; 119:1477-1483.

22 Gameiro GH, Gameiro PH, Andrade AS, et al: Nociception- and anxiety-like behavior in rats submitted to different periods of restraint stress. Physiol Behav 2006;87:643-649.

23 Belzung C, Griebel G: Measuring normal and pathological anxiety-like behaviour in mice: a review. Behav Brain Res 2001;125:141-149.

24 Korte SM, De Boer SF: A robust animal model of state anxiety: fear-potentiated behaviour in the elevated plus-maze. Eur J Pharmacol 2003;463:163-175.

25 Bhattacharya SK, Bhattacharya D: Effect of restraint stress on rat brain serotonin. J Biosci 1982;4:269-274.

26 Cavigelli SA, McClintock MK: Fear of novelty in infant rats predicts adult corticosterone dynamics and an early death. Proc Natl Acad Sci U S A 2003;100:16131-16136. 